Rev. Fac. Agron. (LUZ). 2021, 38: 261-278. Abril-Junio.

DOI: https://doi.org/10.47280/RevFacAgron(LUZ).v38.n2.03

ISSN 2477-9407

\title{
Micropropagation of Pelargonium odoratissimum (L.) L'Her. through petioles and leaves
}

\section{Micropropagación de Pelargonium odoratissimum (L.) \\ L’Her., a través de explantes de hojas y pecíolos}

\section{Micropropagação de Pelargonium odoratissimum L'Her. através de pecíolos e folhas}

Asghar Ebrahimzadeh ${ }^{1, *}$, Maliheh Fathollahzadeh², Mohammad Bagher Hassanpouraghdam ${ }^{3,4}$ and Mohammad Ali Aazami Mavaloo ${ }^{5}$

\begin{abstract}
${ }^{1}$ Assitant Professor, Department of Horticultural Sciences, Faculty of Agriculture, University of Maragheh, Iran. Email:acebrahimzadeh@gmail.com, (D) . ${ }^{2}$ Former Master student, Department of Horticultural Sciences, Faculty of Agriculture, University of Maragheh, Iran. Email: f_malihe@yahoo.com. ${ }^{3}$ Associate Professor, Department of Horticultural Sciences, Faculty of Agriculture, University of Maragheh, Iran. Email: hassanpouraghdam@gmail.com, (D. ${ }^{4}$ Islamic Parliament Research Center, Tehran, Iran. ${ }^{5}$ Assitant Professor, Department of Horticultural Sciences, Faculty of Agriculture, University of Maragheh, Iran. Email: aazami58@gmail.com, (D).
\end{abstract}

\section{Abstract}

Pelargonium odoratissimum (L.) L'Her is a hard rooting plant and the common methods of propagation via stem cuttings are not successful with this species. therefore, tissue culture methods have been experienced for the masspropagation of this high-valued species. Intact leaves, leaf segments and petiole sections derived from nodal explants in vitro were employed for the optimization of $P$. odoratissimum micropropagation. The treatment combinations used were $\mathrm{MS}$ and $1 / 2$ MS media supplemented with 6-benzylaminopurine, $\operatorname{BAP}(1,1.5,2$ and $\left.4.5 \mathrm{mg} . \mathrm{L}^{-1}\right)$ and 1-naphthaleneacetic acid, NAA (0.1, 1 and $\left.1.5 \mathrm{mg} . \mathrm{L}^{-1}\right)$. With leaf segments, the lowest browning incidence, the greatest callogenesis and the highest number of shoots were obtained with the media containing $1.5 \mathrm{mg} . \mathrm{L}^{-1}$ BAP and $1 \mathrm{mg} \cdot \mathrm{L}^{-1} \mathrm{NAA}$. Two mg. $\mathrm{L}^{-1} \mathrm{BAP}+0.1 \mathrm{mg} \cdot \mathrm{L}^{-1} \mathrm{NAA}$ kept the same results for petiole explants. Intact leaves showed the best results for the three mentioned

Recibido el 23-03-2020 • Aceptado el 04-07-2020.

*Correspondig autor. Email: acebrahimzadeh@gmail.com, ebrahimzadeh@maragheh.ac.ir 
Rev. Fac. Agron. (LUZ). 2021, 38: 261-278. Abril-Junio.

Ebrahimzadeh et al.

treatments with $1 \mathrm{mg} \cdot \mathrm{L}^{-1} \mathrm{BAP}+1 \mathrm{mg} \cdot \mathrm{L}^{-1} \mathrm{NAA} .0 .2 \mathrm{mg} \cdot \mathrm{L}^{-1} \mathrm{NAA}$ caused the highest rooting percentage and the greatest mean data for the number and length of the roots. Rooted plantlets were transferred to the pots containing 1:1 peat-moss and perlite. Acclimatization of the plantlets was followed by $90 \%$ of survival rate in the greenhouse. The protocol employed would be a potent one to present for the extension section.

Keyword: nodal explants, MS medium, shoot organogenesis, BAP, NAA, leaf segments.

\section{Resumen}

Pelargonium odoratissimum (L.) L'Her es una planta de complejo enraizamiento y los métodos comunes de propagación mediante esquejes de tallos no presentan un resultado positivo con esta especie. Acorde a lo anterior, han realizado ensayos empleando métodos de cultivo de tejidos para la propagación a gran escala de esta especie de alto valor. Se emplearon: hojas completas, segmentos de hojas y secciones de pecíolo; derivadas de explantes nodales producidos in vitro para la optimización de la micropropagación de P. odoratissimum. Las combinaciones de tratamiento utilizadas fueron: MS y $1 / 2 \mathrm{MS}$ enriquecidos con 6-bencilaminopurina, $\operatorname{BAP}\left(1,1.5,2\right.$ y $\left.4.5 \mathrm{mg} . \mathrm{L}^{-1}\right)$ y ácido naftalenacético, NAA $\left(0.1,1\right.$ y $\left.1.5 \mathrm{mg} . \mathrm{L}^{-1}\right)$. Al utilizar segmentos foliares, se observó menor incidencia de pardeamiento y mayor callogénesis. El mayor número de brotes se obtuvo con el medio de cultivo que contenía 1,5 mg.L ${ }^{-1}$ BAP y $1 \mathrm{mg} . \mathrm{L}^{-1} \mathrm{NAA}$. Los medios de cultivo de $2 \mathrm{mg} . \mathrm{L}^{-1} \mathrm{BAP}+$ $0.1 \mathrm{mg} . \mathrm{L}^{-1} \mathrm{NAA}$, presentaron los mismos resultados para los explantes de peciolo. Las hojas completas mostraron los mejores resultados el emplear el medio de 1 mg.L. ${ }^{-1}$ BAP + 1 mg.L. $\mathrm{L}^{-1}$ NAA. El tratamiento de $0.2 \mathrm{mg} \cdot \mathrm{L}^{-1} \mathrm{NAA}$ causó el mayor porcentaje de enraizamiento y la mayor media de datos para el número y longitud de raíces. Las plántulas enraizadas se transfirieron a materas utilizando como sustrato una mezcla de turba-musgo y perlita, con una relación de mezcla 1:1. El acondicionamiento de las plántulas presentaron una tasa de superviviencia $90 \%$ en el invernadero. El protocolo empleado presento altas tasas de efectividad como medio de propagación para su extensión.

Palabras clave: explantes nodales, MS, organogénesis de brotes, BAP, NAA, segmentos foliares.

\section{Resumo}

Pelargonium odoratissimum (L.) L'Her é uma planta de enraizamento difícil e os métodos comuns de propagação por meio de estacas não têm sucesso com esta espécie. Portanto, métodos de cultura de tecidos têm sido experimentados para a propagação em massa desta espécie de alto valor. Folhas intactas, segmentos foliares e seções de pecíolo derivados de explantes nodais in vitro foram empregados para a otimização da micropropagação de $P$. odoratissimum. As combinações de tratamento utilizadas foram meio MS e $1 / 2$ MS suplementado com 6-benzilaminopurina, $\operatorname{BAP}\left(1,1,5,2\right.$ e 4,5 mg. $\left.\mathrm{L}^{-1}\right)$ e ácido 1-naftalenoacético, NAA 
Rev. Fac. Agron. (LUZ). 2021, 38: 261-278. Abril-Junio.

$\left(0,1,1\right.$ e $\left.1,5 \mathrm{mg} \cdot \mathrm{L}^{-1}\right)$. Nos segmentos foliares, a menor incidência de escurecimento, a maior calogênese e o maior número de brotações foram obtidos com os meios contendo 1,5 mg.L. $\mathrm{L}^{-1}$ de BAP e $1 \mathrm{mg} . \mathrm{L}^{-1}$ de ANA. Dois mg. $\mathrm{L}^{-1}$ de BAP + 0,1 mg.L $\mathrm{L}^{-1}$ de NAA mantiveram os mesmos resultados para explantes de pecíolo. As folhas intactas apresentaram os melhores resultados para os três tratamentos citados com $1 \mathrm{mg} \cdot \mathrm{L}^{-1} \mathrm{BAP}+1 \mathrm{mg} . \mathrm{L}^{-1}$ ANA. $0,2 \mathrm{mg} \cdot \mathrm{L}^{-1} \mathrm{NAA}$ causou a maior porcentagem de enraizamento e os maiores dados médios para o número e comprimento das raízes. As plântulas enraizadas foram transferidas para vasos contendo turfamusgo 1: 1 e perlita. A aclimatação das mudas foi acompanhada por $90 \%$ de sobrevivência em casa de vegetação. O protocolo empregado seria potente para apresentar para a seção de extensão.

Palavras-chave: explantes nodais, meio MS, organogênese caulinar, BAP, NAA, segmentos foliares.

\section{Introduction}

Pelargonium odoratissimum (L.) L'Her. belongs to the Geraniaceae family (Hassanein and Dorion, 2005). Essential oil of this species is widely used in fragrance, hygienic and cosmetic industries. Furthermore, the oil holds antimicrobial, antifungal and insecticidal activities (Gupta et al., 2002). P. odoratissimum is considered a hard-to-root species that is not successfully multiplied when methods of propagation via stem cuttings, commonly used in other Pelargonium species, are applied (Bhuse et al, 2003). Therefore, tissue culture methods have been completed for the mass-propagation of this high-valued species (Gupta et al., 2002). Diverse plant organs have been utilized as micropropagation propagules (Gupta et al., 2002). Shoot organogenesis has been successful with different Pelargonium species via direct (Dunbar and Stephens, 1989) or indirect (callogenesis) procedures (Boase et al., 1998; Agarwal and

\section{Introducción}

\section{Pelargonium}

odoratissimum (L.) L'Her. pertenece a la familia Geraniaceae (Hassanein y Dorion, 2005). El aceite esencial de esta especie es ampliamente utilizado en las industrias de fragancias, higiene y cosméticos. Adicionalmente el aceite, se le atribuye propiedades antimicrobianas, antifúngicas e insecticida (Gupta et al., 2002). Pelargonium odoratissimum se considera una especie de complejo enraizamiento y bajo éxito en los métodos de propagación a través de esquejes de tallo, empleados comúnmente en otras especies de Pelargonium (Bhuse et al, 2003). Esta problemática, ha generado el desarrollo de métodos de cultivos de tejidos para la propagación masiva de esta especie de alto valor industrial (Gupta et al., 2002). Diversos órganos vegetales se han utilizado como propágulos en micropropagación (Gupta et al., 2002). La organogénesis del brote ha tenido éxito con 
Rev. Fac. Agron. (LUZ). 2021, 38: 261-278. Abril-Junio.

Ebrahimzadeh et al.

ISSN 2477-9407

Ranu, 2000). Wojtania et al. (2004) reported the highest shoot organogenesis from petiole segments in 'Bonete' (a hybrid of Pelargonium $\times$ hederaefolium) using a medium containing 2 mg.L $\mathrm{L}^{-1}$ Thidiazuron (TDZ) and $0.1-0.2 \mathrm{mg} . \mathrm{L}^{-1}$ indole3-butyric acid (IBA). Furthermore, shoot proliferation from leaf and petioles of $P$. graveolens was achieved in the Linsmaier and Skoog (LS) medium enriched with $1 \mathrm{mg} . \mathrm{L}$ ${ }^{1}$ of 1-naphthaleneacetic acid (NAA) and $5 \mathrm{mg} . \mathrm{L}^{-1}$ of 6 -benzylaminopurine (BAP) (Ghanem et al., 2008). In another study, Hassanein and Dorion (2005) reported that $1 \mathrm{mg} . \mathrm{L}^{-1}$ Zeatin + $1 \mathrm{mg} . \mathrm{L}^{-1} \mathrm{BAP}+0.5 \mathrm{mg} \cdot \mathrm{L}^{-1} \mathrm{NAA}$ were the growth regulator combination for the highest shoot organogenesis rate in $P$. graveolens cv. "Bois joly". Regarding root organogenesis, the highest rooting percentage was induced on half-strength Murashige y Skoog (MS) medium supplemented with $1 \mathrm{mg} . \mathrm{L}^{-1}$ indolacetic acid (IAA) (Hassanein and Dorion, 2005). Rao (1994) documented that $0.1 \mathrm{mg} . \mathrm{L}^{-1}$ NAA was the best treatment for the optimum rooting treatments in Pelargonium. In another study, 90 $\%$ of the shoots produced roots after 15 days on a medium containing 0.1 mg.L L $^{-1}$ IBA (Tembe and Deodhar, 2010). Moreover, Desilets et al. (1993) reported that the roots emerged easily in a hormone free $1 / 2$ strength MS medium. The main purpose of the present study was to formulate an efficient in vitro propagation protocol for $P$. odoratissimum from leaf and petiole sections and intact leaf. diferentes especies de Pelargonium a través de procedimientos directos (Dunbar y Stephens, 1989) o indirectos (callogénesis) (Boase et al., 1998; Agarwal y Ranu, 2000). Wojtania et al. (2004), reportaron la mayor organogénesis de brotes a partir de segmentos de pecíolos en 'Bonete' (un híbrido de Pelargonium $\mathrm{X}$ hederaefolium) empleando un medio de cultivo de: 2 mg.L ${ }^{-1}$ Tidiazuron (TDZ) y 0,1 - 0,2 mg.L $\mathrm{L}^{-1}$ de acido indobutírico (IBA). Otros estudios, obtuvieron proliferación de brotes de hojas y pecíolos de P. graveolens empleado medio enriquecido de Linsmaier y Skoog (LS) con $1 \mathrm{mg} . \mathrm{L}^{-1}$ acido 1-naftaleacético (NAA) y $5 \mathrm{mg} . \mathrm{L}^{-1}$ 6-benzilaminopurina (BAP) (Ghanem et al., 2008). Hassanein y Dorion (2005) obtuvieron que $1 \mathrm{mg} . \mathrm{L}^{-1}$ Zeatin $+1 \mathrm{mg} \cdot \mathrm{L}^{-1} \mathrm{BAP}+$ 0,5 mg.L $\mathrm{L}^{-1} \mathrm{NAA}$, fue la combinación de reguladores de crecimiento para obtener la tasa más alta de organogénesis de brote en $P$. graveolens cv. "Bois joly". En cuanto a la organogénesis de la raíz, el porcentaje de enraizamiento más alto se indujo con el medio de cultivo Murashige y Skoog (MS) a la mitad de su concentración suplementado con $1 \mathrm{mg} . \mathrm{L}^{-1}$ ácido indolacético (IAA) (Hassanein y Dorion, 2005). Rao (1994) documentó que 0,1 mg. $\mathrm{L}^{-1}$ NAA fue el mejor tratamiento para los tratamientos de enraizamiento óptimos en pelargonio. En otro estudio, el $90 \%$ de los brotes produjeron raíces después de 15 días en un medio con contenido de 0,1 mg.L $\mathrm{L}^{-1}$ IBA (Tembe y Deodhar, 2010). Por otra parte, Desilets et al. (1993) concluyo que las raíces emergieron fácilmente en 
Rev. Fac. Agron. (LUZ). 2021, 38: 261-278. Abril-Junio.

Ebrahimzadeh et al.

ISSN 2477-9407

\section{Materials and methods}

\section{Plant material and superficial sterilization}

Intact leaves, petiole and leaf segments were chosen from in vitro derived shoots. In vitro mother plants were initiated from nodal-cuttings excised from plants grown in a greenhouse. Superficial sterilization of nodal cuttings was completed in a laminar-airflow cabinet as followed: immersion in $70 \%$ ethanol for one minute, followed by sodium hypochlorite $15 \%$ containing 2 drops of Triton X-100 for 13 minutes. Thereafter, the explants were threetimes rinsed in distilled water and then were treated with $70 \%$ alcohol for 20 seconds. The nodal cuttings were cultured in Murashige and Skoog (MS) (Murashige and Skoog, 1962) medium, and after about one month, the new shoots were chosen for the current study explants.

\section{Media and shoot proliferation}

The basal medium was MS supplemented with $3 \%$ sucrose, $0.7 \%$ agar and 500 mg.L. ${ }^{-1}$ myo-inositol. Leaf and petiole segments were cut into 0.5 $\mathrm{cm}$ sections under a laminar airflow cabinet. Intact leaf samples were leaves with about $1 \mathrm{~cm}$ in diameter. The explants were cultured in $1 / 2$ strength MS medium containing $\operatorname{BAP}(1,1.5$, 2 and 4.5 mg.L $\left.\mathrm{L}^{-1}\right)$ and NAA (0.1, 1 and $\left.1.5 \mathrm{mg} . \mathrm{L}^{-1}\right)$ as treatment combinations. To prevent tissue browning $50 \mathrm{mg} \cdot \mathrm{L}^{-1}$ ascorbic acid was added to the medium. Medium pH was adjusted at $5.6-5.7$ before autoclaving at $121{ }^{\circ} \mathrm{C}$ for 20 minutes. Three explants were placed in each $9-\mathrm{cm}$ petri-dish. The cultures un medio de cultivo de $1 / 2$ MS libre de hormona. El objetivo principal del presente estudio fue formular un protocolo de propagación in vitro eficiente para $P$. odoratissimum empleando secciones de hojas y pecíolos, y hojas completas.

\section{Material y métodos}

\section{Material vegetal y esterilización superficial}

Las hojas completas, las secciones de pecíolo y de hojas fueron elegidos de brotes cultivados in vitro. Las plantas madre cultivadas in vitro provienen de cortes nodales de plantas cultivadas en invernadero. La esterilización superficial de esquejes nodales se realizó en una cabina laminar con flujo de aire empleando: inmersión en etanol al $70 \%$ durante un minuto, seguida de hipoclorito sódico $15 \%$ que contiene 2 gotas de Tritón X-100 durante 13 minutos. A partir de entonces, los explantes se limpiaron tres veces en agua destilada y posteriormente fueron tratados con $70 \%$ de alcohol durante 20 segundos. Los esquejes nodales se cultivaron en el medio de Murashige y Skoog (MS) (Murashige y Skoog, 1962), y después de aproximadamente un mes, los nuevos brotes fueron elegidos para los explantes de estudio actuales.

\section{Medio de cultivo y proliferación de brotes}

El medio inicial fue el MS suplementado con $3 \%$ de sacarosa, $0,7 \%$ de agar y $500 \mathrm{mg} \cdot \mathrm{L}^{-1}$ mioinositol. Los segmentos de hojas y pecíolos se cortaron en secciones de $0,5 \mathrm{~cm}$ bajo una cabina de flujo laminar. 
Rev. Fac. Agron. (LUZ). 2021, 38: 261-278. Abril-Junio.

Ebrahimzadeh et al.

ISSN 2477-9407

were placed under dark conditions for two weeks, followed by 16:8 hour photoperiod regime at $23 \pm 1^{\circ} \mathrm{C}$ and $20 \pm$ $1^{\circ} \mathrm{C}$, respectively.

Four weeks after the explants were initiated, crispy and creamy calli developed. Callus formation percentage was measured for each explant. Calli were sub-cultured on the above described medium means MS + all BAP and NAA combinations. Two weeks later, shoots noticed. About two more weeks later, the number and length of the shoots were recorded.

\section{Rooting}

The shoots $(3-4 \mathrm{~cm})$ were excised and placed on $1 / 2$ strength MS medium containing 0.1 or $0.2 \mathrm{mg} . \mathrm{L}^{-1} \mathrm{NAA}$ and 2 mg. $L^{-1}$ active charcoal. The number and length of the roots were recorded two weeks later.

\section{Acclimatization}

After removing the adhered agar from the roots using $50{ }^{\circ} \mathrm{C}$ water, the rooted shoots $(3-4 \mathrm{~cm})$ were transferred to the pots containing 1:1 peat - moss: perlite. Rooted cuttings were placed in growth chambers with 16:8 photoperiod at $23 \pm 1^{\circ} \mathrm{C}$ and $20 \pm$ $1^{\circ} \mathrm{C}$, respectively

\section{Statistical analysis}

This experiment was conducted as factorial experiments arranged in a complete block design with 18 treatment combinations (3 explant types $\times 6$ Plant Growth Regulator (PGR) combinations) with 5 replications in each experimental unit. Each experiment was repeated at least twice and the reported data are the means of two experiments. Data analyses was performed using IBM SPSS software (version 21) (SPSS,
Las muestras de hojas intactas correspondían aproximadamente a hojas de $1 \mathrm{~cm}$ de diámetro. Los explantes se cultivaron en un medio de $1 / 2$ MS que contenía combinaciones de BAP $\left(1 ; 1,5 ; 2\right.$ y 4,5 mg.L. $\left.\mathrm{L}^{-1}\right)$ y NAA $(0,1$; 1 y $\left.1,5 \mathrm{mg} . \mathrm{L}^{-1}\right)$ como tratamientos. Para prevenir el pardeamiento de tejidos, se añadió $50 \mathrm{mg} . \mathrm{L}^{-1}$ de ácido ascórbico al medio. El pH de medio se ajustó a $5,6-5,7$ antes de autoclavado a $121^{\circ} \mathrm{C}$ durante 20 minutos. Se colocaron tres explantes de $9 \mathrm{~cm}$ en cada placa de Petri bajo condiciones de oscuridad durante dos semanas, seguidas por un régimen de fotoperiodo de 16:8 horas a $23 \pm 1^{\circ} \mathrm{C}$ y $20 \pm 1^{\circ} \mathrm{C}$, respectivamente.

Cuatro semanas después de que se iniciaran los explantes, se desarrollaron callos crujientes $\mathrm{y}$ cremosos. Se midió el porcentaje de formación del callo para cada explante. Los callos fueron subcultivados en cada medio de cultivo o tratamiento descrito anteriormente (MS + combinaciones de BAP y NAA). Dos semanas después, se evidenciaron brotes que posteriormente a las dos semanas más tarde, se registraron el número y la longitud de los brotes presentes.

\section{Enraizamiento}

Los brotes (3 - $4 \mathrm{~cm}$ ) fueron extraídos y colocados en un medio de $1 / 2 \mathrm{MS}$ que contiene 0,1 o $0,2 \mathrm{mg} \cdot \mathrm{L}^{-1}$ NAA y $2 \mathrm{mg} . \mathrm{L}^{-1}$ de carbón activado. El número y la longitud de las raíces se registraron dos semanas más tarde.

\section{Aclimatación}

Después de retirar el agar adherido de las raíces usando agua a $50^{\circ} \mathrm{C}$, los brotes enraizados $(3-4 \mathrm{~cm})$ 
Rev. Fac. Agron. (LUZ). 2021, 38: 261-278. Abril-Junio.

Ebrahimzadeh et al.

2012). Mean comparison were carried out by LSD at $5 \%$ probability level.

\section{Results and discussion}

\section{Callogenesis}

Calli developed from the explants incubated in the dark during 4 weeks on $1 / 2$ strength MS medium supplemented with BAP and NAA at different concentration combinations. ANOVA results showed that PGR combinations (BAP + NAA) as well explant type, and their interactions affected the callogenesis, significantly $(p<0.01)$. The results showed that intact leaf explants with $1.5 \mathrm{mg} \cdot \mathrm{L}^{-1}$ BAP +1 mg. $\mathrm{L}^{-1}$ NAA, petiole explants with 2 mg. $\mathrm{L}^{-1} \mathrm{BAP}+0.1 \mathrm{mg} \cdot \mathrm{L}^{-1} \mathrm{NAA}$, all three explant types with $1.5 \mathrm{mg} \cdot \mathrm{L}^{-1} \mathrm{BAP}+1$ mg. $\mathrm{L}^{-1}$ NAA treatment combinations as well as the intact leaf and leaf segments with $4.5 \mathrm{mg} \cdot \mathrm{L}^{-1} \mathrm{BAP}+1$ mg. L $^{-1}$ NAA treatment had maximum callus percentage but there was not significant difference among them (table 1). Therefore, the $1.5 \mathrm{mg} . \mathrm{L}^{-1}$ $\mathrm{BAP}+1 \mathrm{mg} \cdot \mathrm{L}^{-1} \mathrm{NAA}$ combination would be an appropriate treatment for callus proliferation in this species regardless the explant type. Callogenesis is dependent upon the internal and external concentrations of PGRs (Benazir et al., 2013). So, a suitable concentration of auxins and cytokinins is necessary to have reasonable callus formation as initial in vitro propagation stage. In Pelargonium rapaceum, Sukhumpinij et al. (2010) reported that callus production was optimum using leaf explants in a medium se sembraron en macetas con sustrato 1:1 turba - musgo: perlita. Los esquejes enraizados se colocaron en cámaras de crecimiento con fotoperiodo $16: 8$ a $23 \pm$ $1^{\circ} \mathrm{C}$ y $20 \pm 1^{\circ} \mathrm{C}$, respectivamente.

\section{Análisis estadístico}

Este experimento se llevó a cabo como factorial de bloques al azar de 18 tratamientos (3 tipos de explantes $\times 6$ combinaciones de reguladores de crecimiento vegetal (PGR)) con 5 repeticiones en cada unidad experimental. Cada experimento se repitió dos veces y los datos obtenidos son la media de dos experimentos. Los análisis de datos se realizaron utilizando el software IBM SPSS (versión 21) (SPSS, 2012). La comparación media se llevó a cabo por LSD en un nivel de probabilidad del $5 \%$.

\section{Resultados y discusión}

\section{Callogénesis}

Los callos se desarrollaron a partir de los explantes incubados en la oscuridad durante 4 semanas en medio de $1 / 2$ MS suplementado con BAP y NAA en diferentes combinaciones de concentración. Los resultados de ANOVA mostraron que las combinaciones de PGR (BAP + NAA) así como el tipo de explante, $\mathrm{y}$ sus interacciones afectaron a la callogénesis, significativamente $(p<0.01)$. Los resultados mostraron que los explantes de hojas completas con 1,5 mg. $\mathrm{L}^{-1}$ BAP + 1 mg. $\mathrm{L}^{-1} \mathrm{NAA}$, explantes de pecíolo con $2 \mathrm{mg}$. $\mathrm{L}^{-1} \mathrm{BAP}$ $+0,1 \mathrm{mg} \cdot \mathrm{L}^{-1} \mathrm{NAA}$, los tres tipos de explantes con $1.5 \mathrm{mg} \cdot \mathrm{L}^{-1} \mathrm{BAP}+1 \mathrm{mg} \cdot \mathrm{L}^{-1}$ NAA combinaciones de tratamientos, así como los segmentos de hoja y 
Rev. Fac. Agron. (LUZ). 2021, 38: 261-278. Abril-Junio.

Ebrahimzadeh et al.

ISSN 2477-9407

enriched with $0.1 \mathrm{mg} \cdot \mathrm{L}^{-1} \mathrm{BAP}+0.1$ hoja completa con 4,5 mg.L.1 BAP + 1 mg.L ${ }^{-1}$ NAA. Furthermore, Gharib mg.NAA L ${ }^{-1}$ presentaron porcentajes (2005) demonstrated that callus máximos de formación de callos, formation and organogenesis in pero no había diferencia significativa

Table 1. Mean comparison for the effects of explant type and BAP-NAA combinations on callogenesis and shoot organogenesis in Pelargonium odoratissimum.

Cuadro 1. Comparación de los efectos de las combinaciones de tipo de explante y BAP-NAA en la callogénesis y organogénesis de broto en Pelargonium odoratissimum.

\begin{tabular}{|c|c|c|c|c|c|c|}
\hline \multirow[b]{2}{*}{ Explant } & \multicolumn{3}{|c|}{ Study factor } & \multicolumn{3}{|c|}{ Means } \\
\hline & $\begin{array}{l}\text { BAP } \\
\text { mg... }{ }^{-1}\end{array}$ & $\begin{array}{l}\text { NAA } \\
\text { mg.L } L^{-1}\end{array}$ & $\begin{array}{l}\text { Browning } \\
(\%)\end{array}$ & $\begin{array}{c}\text { Callogenesis } \\
(\%)\end{array}$ & $\begin{array}{c}\text { Mean shoot } \\
\text { number }\end{array}$ & $\begin{array}{l}\text { Mean shoot } \\
\text { length }(\mathrm{cm})\end{array}$ \\
\hline Intact leaf & 1 & 1 & $32.3^{\mathrm{efg}}$ & $100^{\mathrm{a}}$ & $15^{\mathrm{ab}}$ & $2.5^{\mathrm{abc}}$ \\
\hline Intact leaf & 1.5 & 1 & $16.3^{g}$ & $100^{\mathrm{a}}$ & $10.6^{\mathrm{cd}}$ & $3^{\mathrm{ab}}$ \\
\hline Intact leaf & 1.5 & 1.5 & $15.3^{g}$ & $86.4^{\mathrm{abc}}$ & $12.5^{\mathrm{bcd}}$ & $3.194^{\mathrm{a}}$ \\
\hline Intact leaf & 2 & 0.1 & $39.9^{\text {def }}$ & $73.6^{\text {bcd }}$ & $8.16^{\mathrm{de}}$ & $2.1^{\mathrm{bcd}}$ \\
\hline Intact leaf & 2 & 1 & $22.9^{\mathrm{fg}}$ & $100^{\mathrm{a}}$ & $13.84^{\mathrm{bc}}$ & $3^{\mathrm{ab}}$ \\
\hline Intact leaf & 4.5 & 1 & $28.6^{\mathrm{efg}}$ & $93.2^{\mathrm{ab}}$ & $8.9^{\text {cde }}$ & $3.46^{\mathrm{a}}$ \\
\hline Leaf segments & 1 & 1 & $71^{\mathrm{abc}}$ & $39.6^{\mathrm{fg}}$ & $2.1^{\mathrm{f}}$ & $0.9^{\mathrm{ef}}$ \\
\hline Leaf segments & 1.5 & 1 & $12.3^{\mathrm{g}}$ & $100^{\mathrm{a}}$ & $19.92^{\mathrm{a}}$ & $3^{\mathrm{ab}}$ \\
\hline Leaf segments & 1.5 & 1.5 & $73^{\mathrm{abc}}$ & $36^{\mathrm{fg}}$ & $2^{\mathrm{f}}$ & $0.55^{\mathrm{ef}}$ \\
\hline Leaf segments & 2 & 0.1 & $42.64^{\mathrm{def}}$ & $79^{\mathrm{abcd}}$ & $9.16^{\text {cde }}$ & $2.5^{\mathrm{abc}}$ \\
\hline Leaf segments & 2 & 1 & $46.5^{\mathrm{dc}}$ & $66.58^{\text {cde }}$ & $5.29^{\mathrm{ef}}$ & $2^{\text {cd }}$ \\
\hline Leaf segments & 4.5 & 1 & $33.3^{\text {efg }}$ & $100^{\mathrm{a}}$ & $11.7^{\mathrm{bcd}}$ & $2.42^{\mathrm{abc}}$ \\
\hline petiole & 1 & 1 & $58.9^{\mathrm{bcd}}$ & $73 b^{c d}$ & $4.36^{\mathrm{ef}}$ & $1.2^{\mathrm{def}}$ \\
\hline petiole & 1.5 & 1 & $40.6^{\text {def }}$ & $93.2^{\mathrm{ab}}$ & $2.78^{\mathrm{f}}$ & $1.35^{\mathrm{de}}$ \\
\hline petiole & 1.5 & 1.5 & $79.3^{\mathrm{ab}}$ & $45.6^{\mathrm{efg}}$ & $1.78^{\mathrm{f}}$ & $0.456^{\mathrm{ef}}$ \\
\hline petiole & 2 & 0.1 & $15.3^{\mathrm{g}}$ & $100^{\mathrm{a}}$ & $19.1^{\mathrm{a}}$ & $3.3^{\mathrm{a}}$ \\
\hline petiole & 2 & 1 & $92.6^{\mathrm{a}}$ & $25.32^{\mathrm{g}}$ & $0.66^{\mathrm{f}}$ & $0.21^{\mathrm{f}}$ \\
\hline petiole & 4.5 & 1 & $56.38^{\mathrm{cd}}$ & $59.6^{\text {def }}$ & $2^{\mathrm{f}}$ & $1.12^{\text {ef }}$ \\
\hline
\end{tabular}

Different letters indicate significant difference within each column based on LSD test at $\mathrm{p}<0.05$; NAA = 1-naphthaleneacetic acid; BAP = 6-Benzylaminopurine.

Diferentes letras indican una diferencia significativa dentro de cada columna basada en la prueba de LSD en $\mathrm{p}<0.05 ; \mathrm{NAA}=1$-Acido naftalenacético; $\mathrm{BAP}=6$-Benzilaminopurina. 
Rev. Fac. Agron. (LUZ). 2021, 38: 261-278. Abril-Junio.

Ebrahimzadeh et al.

$P$. nervosum occurred on a medium supplemented with $5 \mathrm{mg} \cdot \mathrm{L}^{-1} \mathrm{BAP}+$ $1 \mathrm{mg} . \mathrm{L}^{-1}$ NAA.

For the prevention of explant browning in vitro, the culture media was supplemented with 50 mg. $L^{-1}$ ascorbic acid. Desilets et al. (1993) reported that in $P$. hortorum, browning was reduced by using half concentration of macro-nutrients and lower concentration than $2.2 \mathrm{mg} \cdot \mathrm{L}^{-1}$ of BAP and NAA. Table (1) shows that the lowest browning rate in leaf segments was achieved in the medium containing 1.5 and $4.5 \mathrm{mg} \cdot \mathrm{L}^{-1} \mathrm{BAP}+$ $1 \mathrm{mg} . \mathrm{L}^{-1} \mathrm{NAA}$ with petiole, $2 \mathrm{mg} . \mathrm{L}^{-1}$ $\mathrm{BAP}+0.1 \mathrm{mg} \cdot \mathrm{L}^{-1} \mathrm{NAA}$ and, in intact leaf samples all the BAP and NAA combinations excluding $2 \mathrm{mg} \cdot \mathrm{L}^{-1}$ $\mathrm{BAP}+0.1 \mathrm{mg} \cdot \mathrm{L}^{-1} \mathrm{NAA}$ had the least percentage of explants with browning. In short, results showed that the intact leaf as well leaf segments slightly affected by oxidation process, whereas, petiole explants was more susceptible to oxidation. Arshad et al. (2012) reported that the highest survival rate in $P$. capitatum was obtained in the medium containing 2 mg. $\mathrm{L}^{-1} \mathrm{BA}+1$ or $2 \mathrm{mg} . \mathrm{L}^{-1}$ NAA. Saxena et al. (2000) reported that using 0.5 mg. $\mathrm{L}^{-1}$ BAP instead of kinetin +0.1 mg. $L^{-1}$ NAA increased the survival rate in $P$. graveolens leaf explants.

Conversely, 1,5 and $2 \mathrm{mg} \cdot \mathrm{L}^{-1}$ $\mathrm{BAP}+1,5$ and $1 \mathrm{mg} \cdot \mathrm{L}^{-1} \mathrm{NAA}$ with petiole explants and 1 and 1,5 mg.L${ }^{1}$ BAP and NAA with leaf segments possessed the highest browning rate and hence, imposed negative effects on the growth and development of shoots and greatly reduced the survival rate. Overall, with all used PGR entre ellos (cuadro 1). Por lo tanto, el 1,5 mg.L $\mathrm{L}^{-1}$ BAP +1 mg.L $\mathrm{L}^{-1} \mathrm{NAA}$ combinación sería un tratamiento adecuado para la proliferación de callos en esta especie independientemente del tipo del explante. La callogénesis depende de las concentraciones internas y externas de los PGR (Benazir et al., 2013). Por lo tanto, una concentración adecuada de auxinas y citoquinas es necesaria para tener una formación razonable de callos como etapa inicial de propagación in vitro. En Pelargonium rapaceum, Sukhumpinij et al. (2010) informaron que la producción de callos era óptima utilizando explantes de hojas en un medio enriquecido con $0,1 \mathrm{mg} . \mathrm{L}^{-1} \mathrm{BAP}$ $+0,1 \mathrm{mg} \cdot \mathrm{L}^{-1}$ NAA. Además, Gharib (2005) demostró que la formación del callos y la organogénesis en $P$. nervosum ocurrieron en un medio complementado con 5 mg. $\mathrm{L}^{-1} \mathrm{BAP}+1$ mg. $L^{-1}$ NAA.

Para la prevención del pardeamiento en los explantes in vitro, los medios de cultivo se complementaron con 50 mg.L $\mathrm{L}^{-1}$ de ácido ascórbico. Desilets et al. (1993) informaron que en $P$. hortorum, el pardeamiento se redujo mediante el uso de la concentración media de macronutrientes y una concentración menor que 2,2 mg. $\mathrm{L}^{-1}$ de BAP y NAA. El cuadro (1) muestra que la tasa de pardeamiento más baja en los segmentos de hoja se alcanzó en el medio que contiene 1,5 y 4,5 mg.L $\mathrm{L}^{-1} \mathrm{BAP}+1$ mg. $\mathrm{L}^{-1} \mathrm{NAA}$ con pecíolo, $2 \mathrm{mg} . \mathrm{L}^{-1} \mathrm{BAP}+0,1 \mathrm{mg} . \mathrm{L}^{-1} \mathrm{NAA}$ $\mathrm{y}$, en las muestras de hojas completas, todas las combinaciones de BAP y NAA, excepto 2 mg.L $\mathrm{L}^{-1} \mathrm{BAP}+0,1$ mg. $L^{-1}$ NAA tenía el menor porcentaje 
Rev. Fac. Agron. (LUZ). 2021, 38: 261-278. Abril-Junio.

Ebrahimzadeh et al.

combinations, intact leaf samples had the highest survival rate. This is the first report of using intact leaves from in vitro propagules. It seems that the great callus proliferation potential and survival rate of intact leaf might be related internal hormonal balance, the lack of injure to the leaf blade and hence, the low incidence of oxidation of phenolic compounds (Skoog and Miller, 1957).

\section{Shoot proliferation}

ANOVA results revealed that the shoot mean number was affected by treatments $(\mathrm{p}<0.01)$, but shoot mean length was not significantly affected by them. For shoot proliferation (table 1), the calli derived from all the explants were sub-cultured on $1 / 2$ MS medium supplement by BAP and NAA combinations. After two weeks, developing shoots were noticeable. The responses in shoot production were different for the diverse treatment combinations. Petiole explants cultured on medium containing $2 \mathrm{mg} . \mathrm{L}^{-1}$ BAP +0.1 mg. $\mathrm{L}^{-1} \mathrm{NAA}$, intact leaf on 1 mg. $\mathrm{L}^{-1} \mathrm{BAP}+1 \mathrm{mg} \cdot \mathrm{L}^{-1} \mathrm{NAA}$ and leaf segments grown on $1.5 \mathrm{mg} . \mathrm{L}^{-1} \mathrm{BAP}$ $+1 \mathrm{mg} \cdot \mathrm{L}^{-1} \mathrm{NAA}$ had the significantly highest shoot numbers (19.1, 15, and 19.92, respectively). No significant differences were determined among these treatments. Hassanein and Dorion (2005) reported the highest shoot number from the leaf segments of $P$. capitatum and $P$. graveolens cultured in a $1 / 2$ MS medium containing $0.5 \mathrm{mg} . \mathrm{L}^{-1} \mathrm{NAA}+1 \mathrm{mg} . \mathrm{L}^{-1} \mathrm{BAP}$ and zeatin. Zhou et al. (2007) noted that leaf segments and petiole explants produced the high number of shoots in a medium enriched with $1 \mathrm{mg} \cdot \mathrm{L}^{-1}$ de explantes con pardeamiento. En resumen, los resultados mostraron que los segmentos de hojas completas, así comolos segmentos de hoja ligeramente afectados por el proceso de oxidación, mientras que, los explantes de pecíolo eran más susceptibles a la oxidación. Arshad et al. (2012) informaron que la tasa de supervivencia más alta en $P$. capitatum se obtuvo en el medio que contiene $2 \mathrm{mg} \cdot \mathrm{L}^{-1} \mathrm{BA}+1$ o $2 \mathrm{mg} \cdot \mathrm{L}^{-1}$ NAA. Saxena et al. (2000) informaron que usando 0,5 mg. $\mathrm{L}^{-1} \mathrm{BAP}$ en lugar de kinetina $+0,1 \mathrm{mg}$. $\mathrm{L}^{-1} \mathrm{NAA}$ aumentó la tasa de supervivencia en los explantes de hojas de $P$. graveolens.

Por el contrario, 1,5 y 2 mg. $\mathrm{L}^{-1}$ BAP +1.5 y 1 mg. $\mathrm{L}^{-1} \mathrm{NAA}$ con explantes de pecíolo y 1 y $1,5 \mathrm{mg} . \mathrm{L}^{-1}$ BAP y NAA con segmentos de hoja presentaron la tasa de pardeo más alta y por lo tanto, efectos negativos en el crecimiento y desarrollo de brotes y redujo en gran medida la tasa de supervivencia. En general, con todas las combinaciones de PGR usadas, las muestras de hojas completas tenían la tasa de supervivencia más alta. Este es el primer informe de uso de hojas completas en la propagación in vitro. Parece que el gran potencial de proliferación del callo y la tasa de supervivencia de la hoja intacta podrían estar relacionados con el equilibrio hormonal interno, la falta de daño a la hoja y, por lo tanto, la baja incidencia de oxidación de compuestos fenólicos (Skoog y Miller, 1957).

\section{Proliferación de brotes}

Los resultados de ANOVA revelaron que el promedio de brotes se vió afectado por los tratamientos 
BAP + 1 mg.L - $^{-1}$ NAA. However, due to our results, it seems that intact leaves yielded more mean shoot number in proliferation of $P$. odoratissimum. According to the findings of Zuraida et al. (2013), using 3-5 mg.L. $\mathrm{L}^{-1}$ BAP reduced the shoot proliferation in Pelargonium species. Therefore, for the suitable proliferation, we need a defined concentration of cytokinins. According to table 1 petiole developed the shortest shoots on all PGR combinations except $2 \mathrm{mg} \cdot \mathrm{L}^{-1} \mathrm{BAP}+0.1$ mg. $L^{-1}$ NAA, shoots from leaf segments with 1.5, 2 and $4.5 \mathrm{mg} . \mathrm{L}^{-1} \mathrm{BAP}+0.1$ and 1 mg.L. $\mathrm{L}^{-1} \mathrm{NAA}$ and intact leaf samples in all treatment combinations except $2 \mathrm{mg} \cdot \mathrm{L}^{-1} \mathrm{BAP}+0.1 \mathrm{mg} \cdot \mathrm{L}^{-1} \mathrm{NAA}$ had tallest shoots, with no significant differences among them. Accordingly, the longest shoots in leaf sections and petiole of $P$. odoratissimum belonged to the medium containing $1 \mathrm{mg} \cdot \mathrm{L}^{-1}$ $\mathrm{NAA}+5 \mathrm{mg} \cdot \mathrm{L}^{-1} \mathrm{BAP}$ (Ghanem et al., 2008).

Brown and Charlwood (1986) demonstrated that the induction and subsequent elongation of adventitious shoots were dependent upon the different factors such as type and concentration of PGRs especially auxins and cytokinins. Therefore, high cytokinin to auxin ratio is the factor that affects the proliferation and shoot length in Pelargonium species (Brown and Charlwood, 1986; Dunbar and Stephens, 1989). High cytokinin and low auxin rates led to increased shoot formation and elongation. Auxin endogenous rates surely affect the phenomenon. It has been reported by former authors where they noted that high cytokinin rates improves the $(p<0,01)$, pero la longitud promedio del brote no se afecta significativamente por ellos. Para la proliferación de brotes (cuadro 1), los callos derivadas de todos los explantes fueron subcultivados en suplemento medio de $1 / 2$ MS por combinaciones de BAP y NAA. Después de dos semanas, los desarrollos de brotes se notaron. Las respuestas en la producción de brotes fueron diferentes para las diversas combinaciones de tratamiento. Los explantes de pecíolo cultivado en medio de cultivo que contenian $2 \mathrm{mg} \cdot \mathrm{L}^{-1} \mathrm{BAP}$ $+0,1 \mathrm{mg} \cdot \mathrm{L}^{-1} \mathrm{NAA}$, hoja completa y 1 mg. $\mathrm{L}^{-1} \mathrm{BAP}+1 \mathrm{mg} \cdot \mathrm{L}^{-1} \mathrm{NAA}$ y segmentos de hoja cultivados y $1.5 \mathrm{mg} . \mathrm{L}^{-1} \mathrm{BAP}+$ $1 \mathrm{mg} . \mathrm{L}^{-1} \mathrm{NAA}$ presentaron el número de brote significativamente más altos (19,1; 15 y 19,92, respectivamente). No se determinaron diferencias significativas entre estos tratamientos. Hassanein y Dorion (2005) informaron el número de brotes más alto de los segmentos de hojas de $P$. capitatum y $P$. graveolens cultivadas en un medio de $1 / 2$ MS que contenia $0,5 \mathrm{mg} \cdot \mathrm{L}^{-1} \mathrm{NAA}+1$ mg.L. ${ }^{-1}$ BAP y zeatin. Zhou et al. (2007) señalaron que los segmentos de hojas y explantes de pecíolos producían el alto número de brotes en un medio de cultivo enriquecido con $1 \mathrm{mg} . \mathrm{L}^{-1}$ BAP $+1 \mathrm{mg}$. $\mathrm{L}^{-1}$ NAA. Sin embargo, debido a nuestros resultados, parece que las hojas intactas dieron más número medio de brotes en la proliferación de $P$. odoratissimum. Según las conclusiones de Zuraida et al. (2013), utilizando 3 - 5 mg. $\mathrm{L}^{-1}$ BAP redujo la proliferación de brotes en especies de Pelargonium. Se puede inferir que, para la proliferación adecuada, es necesaria una concentración definida 
Rev. Fac. Agron. (LUZ). 2021, 38: 261-278. Abril-Junio.

Ebrahimzadeh et al.

proliferation and shoot organogenesis. Moreover, high organogenesis potential in Pelargonium species has been reported by using different explants like leaf and petioles (Dunbar and Stephens, 1989; Yi Qy et al., 2010).

In our experiment, leaf segments with $1.5 \mathrm{mg} . \mathrm{L}^{-1} \mathrm{BAP}+1 \mathrm{mg} . \mathrm{L}^{-1} \mathrm{NAA}$, petioles with $2 \mathrm{mg} \cdot \mathrm{L}^{-1} \mathrm{BAP}+0.1 \mathrm{mg} \cdot \mathrm{L}^{-1}$ NAA and intact leaves with $1 \mathrm{mg} \cdot \mathrm{L}^{-1}$ $\mathrm{BAP}+1 \mathrm{mg} \cdot \mathrm{L}^{-1} \mathrm{NAA}$ achieved the lowest rate of browning, the highest callus production with the subsequent greatest shoot number and tallest shoots.

\section{Rooting and acclimatization}

ANOVA (table 2) results revealed that the rooting related characteristics such as rooting percentage and mean number and length of roots were affected by NAA concentration $(p<0.01)$. Furthermore, mean number and length of roots were significantly $(p<0.01)$ affected by explant type. Just de citoquinas. Según la cuadro 1, pecíolo desarrollado los brotes más cortos en todas las combinaciones de PGR excepto $2 \mathrm{mg} \cdot \mathrm{L}^{-1} \mathrm{BAP}+0,1$ mg. $L^{-1}$ NAA, brotes de segmentos de hoja con 1,5; 2 y 4,5 mg.L.-1 BAP + 0,1 y $1 \mathrm{mg} \cdot \mathrm{L}^{-1}$ NAA y muestras de hojas intactas en todas las combinaciones de tratamiento excepto $2 \mathrm{mg} . \mathrm{L}^{-1} \mathrm{BAP}$ $+0,1 \mathrm{mg} \cdot \mathrm{L}^{-1} \mathrm{NAA}$, tuvo los brotes más altos, sin diferencias significativas entre ellos. En consecuencia, los brotes más largos en secciones de hojas y pecíolos de $P$. odoratissimum pertenecían al medio de cultivo que contenía $1 \mathrm{mg} \cdot \mathrm{L}^{-1} \mathrm{NAA}+5 \mathrm{mg} \cdot \mathrm{L}^{-1} \mathrm{BAP}$ (Ghanem et al., 2008).

Brown y Charlwood

(1986) demostraron que la inducción $y$ posterior elongación de brotes adventicios dependían de los diferentes factores como el tipo y la concentración de PGR, especialmente auxinas y citoquinas. Por lo tanto,

\section{Table 2. ANOVA for the effects of NAA on roots characteristics of Pelargonium odoratissimum explants.}

\section{Cuadro 2. ANOVA para los efectos de NAA en las características de raíces de explantes de Pelargonium odoratissimum.}

Mean Squares

\begin{tabular}{ccccc}
\hline Source of variation & DF & Rooting $(\%)$ & Mean root number & Mean root length \\
NAA & 1 & $267.27^{* *}$ & $248.88^{* *}$ & $9.36^{* *}$ \\
Explant types & 2 & $1.16^{\mathrm{ns}}$ & $39.04^{* *}$ & $1.72^{* *}$ \\
NAA $\times$ Explant types & 2 & $41.31^{\mathrm{ns}}$ & $6.25^{\mathrm{ns}}$ & $0.83^{*}$ \\
error & & 81.32 & 3.21 & 0.27 \\
CV & & 12.56 & 32.37 & 21.86 \\
\hline
\end{tabular}

\footnotetext{
** Significant at $\mathrm{p}<0.01,{ }^{*}$ significant at $\mathrm{p}<0.05$ and ns: non-significant. NAA $=1$-naphthaleneacetic acid.

** Significativo en $\mathrm{p}<0,01 ; *$ significativo en $\mathrm{p}<0,05$ y ns: no significativo. NAA $=$ Acido 1-naftalenacético.
} 
Rev. Fac. Agron. (LUZ). 2021, 38: 261-278. Abril-Junio.

Ebrahimzadeh et al.

root length was influenced $(\mathrm{p}<0.05)$ by the interactions of treatment and explant type.

Figure 1 shows that rooting percentage of the shoots derived from leaf segments, petioles and intact leaves was about $58 \%$ and $98 \%$ at 0.1 and $0.2 \mathrm{mg} . \mathrm{L}^{-1} \mathrm{NAA}$, respectively. NAA concentration suitable for rooting for all three explants was 0.2 mg. $\mathrm{L}^{-1}$ Boase et al. (1998), Zhou et al. (2007) and Zuraida et al. (2013) reported the same NAA concentration for the best rooting percentage and root number in Pelargonium species as well. Brown and Charlwood (1986) as well as Ghanem et al. (2008) also reported that for $P$. graveolens. For root number, $0.2 \mathrm{mg} . \mathrm{L}^{-1} \mathrm{NAA}$ was the best concentration. Among the explants, intact leaves had the highest mean root number (table 3 ). la alta proporción de citoquina a auxina es el factor que afecta a la proliferación y la longitud del brote en especies de Pelargonium (Brown y Charlwood, 1986; Dunbar y Stephens, 1989). Las altas de citoquina y bajas tasas de auxina indujeron un aumento de la formación y elongación del brote. Las tasas endógenas de auxina seguramente afectan el fenómeno. Algunos autores al observado que altas tasas de citoquinas mejora la proliferación y organogénesis del brotes. Además, se ha notificado un alto potencial de organogénesis en especies de Pelargonium mediante el uso de diferentes explantes como hojas y pecíolos (Dunbar y Stephens, 1989; Yi Qy et al., 2010). En nuestro experimento, segmentos de hoja con 1,5 mg. $\mathrm{L}^{-1}$ BAP +1 mg.L $\mathrm{L}^{-1}$ NAA, pecíolos con 2 mg.L. ${ }^{-1}$ BAP +

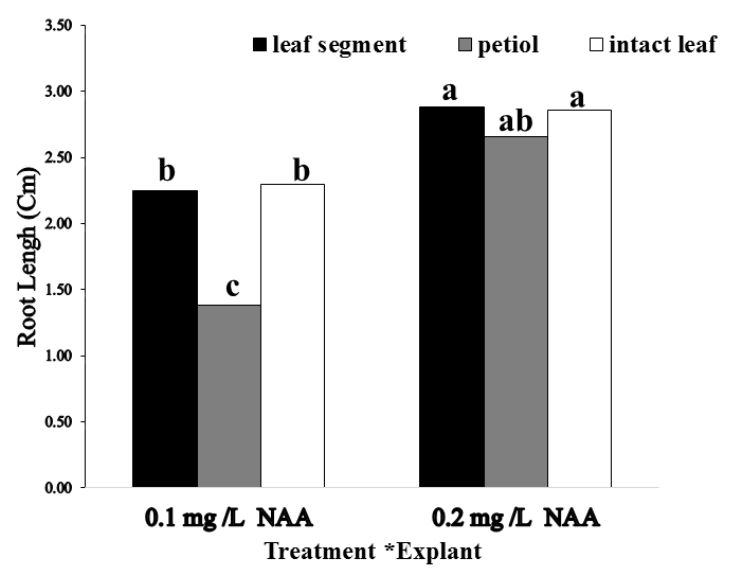

Figure 1. Effect of NAA concentration on the percentage of rooting of Pelargonium odoratissimum in vitro. NAA = 1-naphthaleneacetic acid.

Figura 1. Efecto de concentración de NAA en el porcentaje de enraizamiento de Pelargonium odoratissimum in vitro. NAA = 1-ácido naftalenacético. 
Rev. Fac. Agron. (LUZ). 2021, 38: 261-278. Abril-Junio.

Ebrahimzadeh et al.

ISSN 2477-9407

Table 3. NAA concentration and explant type effects on root number of Pelargonium odoratissimum in vitro.

Cuadro 3. Efectos de concentración de NAA y tipo de explante en el número de raíces de Pelargonium odoratissimum in vitro.

\begin{tabular}{cc}
\hline NAA concentrations and explant types & Root number \\
\hline 0.1 mg.L - $^{-1} \mathrm{NAA}$ & $3.50^{\mathrm{b}}$ \\
0.2 mg.L $\mathrm{L}^{-1} \mathrm{NAA}$ & $7.57^{\mathrm{a}}$ \\
Leaf segments & $4.82^{\mathrm{b}}$ \\
Petiole & $4.64^{\mathrm{b}}$ \\
Intact leaf & $7.15^{\mathrm{a}}$ \\
\hline
\end{tabular}

Different letters show statistically significant differences based on LSD test at $\mathrm{p}<0.05$.

Letras diferentes muestran diferencias estadísticas significativas basadas en la prueba de LSD en $\mathrm{p}<0,05$.

As an accepted rule in tissue culture studies, auxin alone or in combination with small ratios of cytokinins are necessary for the root primordial initiation (Zuraida et al., 2013). Internal hormonal balance is another major factor that affects the rooting potential of different explants. Figure 2 also clearly shows that for all explant types, again $0.2 \mathrm{mg} . \mathrm{L}^{-1} \mathrm{NAA}$ was the best concentration for the length of roots. Boase et al. (1998) reported that increasing NAA concentration up to 0.2 mg. $L^{-1}$ improved root formation in $P$. odoratissimum. Several former studies claim that rooting potential in Pelargonium species is related to the shoots quality.

Zuraida et al. (2013) showed that the highest rooting percentage and number was obtained in a medium enriched with $0.2 \mathrm{mg} \cdot \mathrm{L}^{-1} \mathrm{IBA}+\mathrm{IAA}$. Similarly, Zhou et al. (2007) reported the same concentration of NAA for the rooting of Pelargonium plants. As all physiological processes, rooting potential and its subsequent growth
0,1 mg.L $\mathrm{L}^{-1}$ NAA y hojas completas con $1 \mathrm{mg} \cdot \mathrm{L}^{-1} \mathrm{BAP}+1 \mathrm{mg} \cdot \mathrm{L}^{-1} \mathrm{NAA}$ presentaron la tasa más baja de pardeamiento, la mayor producción de callos con el mayor número de brotes subsiguiente y los brotes más altos.

\section{Enraizamiento y aclimatación}

Los resultados de ANOVA (Cuadro 2) revelaron que las características relacionadas con el enraizamiento, como el porcentaje de enraizamiento y el número medio y la longitud de las raíces se vieron afectadas por la concentración de NAA $(p<0,01)$. Además, el número y la longitud de las raíces se vieron significativamente $(p<0,01)$ afectados por el tipo de explante. La longitud de la raíz fue influenciada $(\mathrm{p}<0,05)$ por las interacciones del tratamiento y el tipo de explante.

La figura (1) muestra que el porcentaje de enraizamiento de los brotes derivados de segmentos foliares, pecíolos y hojas completas fue de aproximadamente el $58 \%$ y el $98 \%$ en 0,1 y 0,2 mg.L. ${ }^{-1}$ NAA, respectivamente. La concentración de 
Rev. Fac. Agron. (LUZ). 2021, 38: 261-278. Abril-Junio.

Ebrahimzadeh et al.

and development are dependent on hormonal balance inside the plant tissue. It seems that in some plant organs, the low amount of internal auxins necessitates the use of compensating concentration of exogenous auxins.
NAA adecuada para el enraizamiento de los tres explantes fue de 0,2 mg.L $\mathrm{L}^{-1}$. Boase et al. (1998), Zhou et al. (2007) y Zuraida et al. (2013) informaron también la misma concentración de NAA para el mejor porcentaje de enraizamiento y el número de raíces

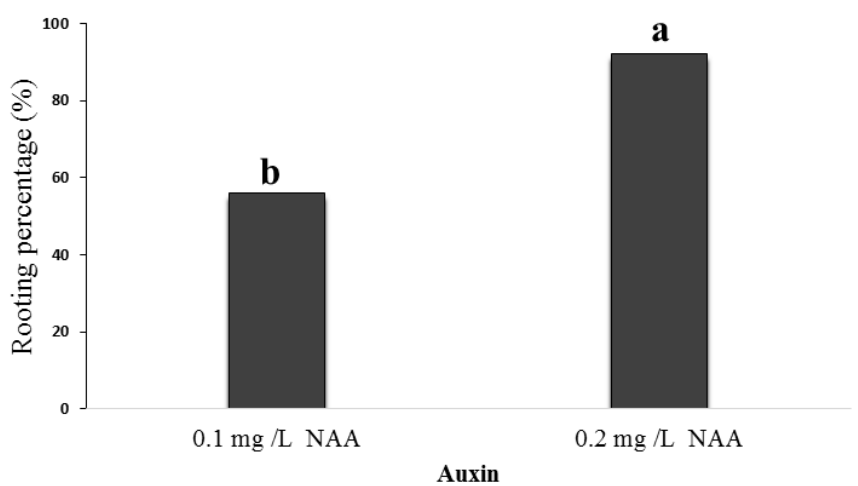

Figure 2. NAA concentration effect on root length of Pelargonium odoratissimum in vitro. $\mathrm{NAA}=1$-naphthaleneacetic acid.

Figura 2. Efecto de concentración de NAA sobre la longitud de la raíz de Pelargonium odoratissimum in vitro. $\mathrm{NAA}=1$-ácido naftalenacético.

Generally, root number and length affect the nutrients and water uptake and hence, hugely influence the plantlets establishment and growth. Finally, healthy rooted plantlets were selected to transfer to the pots containing peat-moss: perlite (1:1) in growth chambers under 16:8 hour photoperiod under $23 \pm 1{ }^{\circ} \mathrm{C}$ and $20 \pm 1{ }^{\circ} \mathrm{C}$ regime. One month later, the number of survived plants were recorded. Acclimatization was completely successful with $90 \%$ of transferred plants were adapted to the greenhouse conditions. en las especies de Pelargonium. Brown y Charlwood (1986) así como Ghanem et al. (2008) también informaron que en $P$. graveolens. para el número de raíz, 0,2 mg.L $\mathrm{L}^{-1}$ NAA fue la mejor concentración. Entre los explantes, las hojas completas tenían el número medio más alto de raíces (cuadro 3).

Como regla aceptada en los estudios de cultivo de tejidos, la auxina sola o en combinación con pequeñas proporciones de citoquinas son necesarias para la iniciación primordial de la raíz (Zuraida et 
Rev. Fac. Agron. (LUZ). 2021, 38: 261-278. Abril-Junio.

Ebrahimzadeh et al.

\section{Conclusion}

This study was conducted to establish an efficient micropropagation protocol for $P$. odoratissimum. The overall results showed that in vitro derived intact leaf explants were superior to the other two explants (petiole and leaf segments), when considering their higher callus, shoot and root organogenesis and proliferation potential. Nonetheless, the BAP-NAA combination rates determined to alarge extent the organogenesis potential. The highest callogenesis and shoot organogenesis were recorded for intact leaves on MS medium supplemented with $1 \mathrm{mg} \cdot \mathrm{L}^{-1}$ BAP +1 mg.L. $\mathrm{L}^{-1} \mathrm{NAA}$, with similar results for leaf segments when grown on 1.5 and 1 mg.L $\mathrm{L}^{-1}$ BAP and NAA, respectively, whereas petioles required 2 mg.L $\mathrm{L}^{-1}$ BAP and $0.1 \mathrm{mg} . \mathrm{L}^{-1} \mathrm{NAA}$ to bring out similar organogenesis. Acclimatization was $90 \%$ successful under greenhouse conditions.

\section{End of English Version}

al., 2013). El equilibrio hormonal interno es otro factor importante que afecta el potencial de enraizamiento de diferentes explantes. La figura 2 también muestra claramente que para todos los tipos de explantes, de nuevo 0,2 mg. $\mathrm{L}^{-1} \mathrm{NAA}$ fue la mejor concentración para la longitud de las raíces. Boase et al. (1998) informaron que el aumento de la concentración de NAA hasta 0,2 mg. $\mathrm{L}^{-1}$ mejoró la formación de raíces en $P$. odoratissimum. Varios estudios anteriores afirman que el potencial de enraizamiento en especies de Pelargonium está relacionado con la calidad de los brotes.

Zuraida et al. (2013) mostraron que el mayor porcentaje y número de enraizamiento se obtuvo en un medio de cultivo enriquecido con 0,2 mg.L ${ }^{-1}$ IBA + IAA. Del mismo modo, Zhou et al. (2007) informaron de la misma concentración de NAA para el enraizamiento de plantas de Pelargonium. Como todos los procesos fisiológicos, potencial de enraizamiento y su posterior crecimiento y desarrollo dependen del equilibrio hormonal dentro del tejido vegetal. Parece que, en algunos órganos vegetales, la baja cantidad de auxinas internas requiere el uso de la concentración compensadora de auxinas exógenas.

Generalmente, el número de raíces y la longitud afectan los nutrientes y la absorción de agua y, por lo tanto, influyen enormemente en el establecimiento y crecimiento de las plántulas. Finalmente, se seleccionaron plántulas de raíces sanas para trasplantar a las materas que contienen sustrato de turba-musgo: perlita (1:1) en cámaras de crecimiento inferiores a 16:8 horas de fotoperiodo bajo $23 \pm$ régimen de $1^{\circ} \mathrm{C}$ y $20 \pm 1^{\circ} \mathrm{C}$. Un mes más tarde, se registró el número de plantas desarrolladas exitosamente. $\mathrm{El}$ acondicionamiento presento valores altamente significativos, el $90 \%$ de las plantas transferidas se adaptaron a las condiciones de invernadero.

\section{Conclusión}

Este estudio se llevó a cabo para establecer un protocolo de 
Rev. Fac. Agron. (LUZ). 2021, 38: 261-278. Abril-Junio.

Ebrahimzadeh et al.

micropropagación eficiente para $P$. odoratissimum. Los resultados generales mostraron que los explantes de hojas completas derivados in vitro eran superiores a los otros dos explantes (pecíolo y segmentos foliares), al considerar su mayor potencial en el desarrollo de la callosidad, brote, organogénesis de raíz y proliferación. No obstante, las tasas de combinación BAP-NAA determinaron en gran medida el potencial de organogénesis. Los datos de mayor resultado en desarrollo de callogénesis y organogénesis de brotes se registraron para las hojas completas en el medio de cultivo MS suplementado con $1 \mathrm{mg} \cdot \mathrm{L}^{-1} \mathrm{BAP}+1$ mg.L. ${ }^{-1}$ NAA, con resultados similares para segmentos de foliares cuando se cultiva en 1.5 y 1 mg. $\mathrm{L}^{-1}$ BAP y NAA, respectivamente, mientras que los pecíolos requirió $2 \mathrm{mg} \cdot \mathrm{L}^{-1}$ BAP y $0.1 \mathrm{mg} . \mathrm{L}^{-1} \mathrm{NAA}$ para sacar a la salida organogénesis similar. El acondicionamiento tuvo éxito en un $90 \%$ en condiciones de invernadero.

\section{Cited literature}

Agarwal, R.K. and A.S. Ranu. 2000. Regeneration of plantlets from leaf and petiole explants of Pelargonium hortorum. In Vitro Cell. Dev. Biol. Plant. 36:392-397.

Arshad, M., J. Silvestre, G. Merlina, C. Dumat, E. Pinelli and J. Kellerhoff. 2012. Thidiazuroninduced shoot organogenesis from mature leaf explants of scented Pelargonium capitatum cultivars. Plant. Cell. Tiss. Org. Cult. 108: 315-322.

Benazir, J.F., R. Suganthi, P. Chandrika and B. Mathithumilan. 2013. In vitro regeneration and transformation studies on Pelargonium graveolens
(Geranium) an important medicinal and aromatic plant. J. Med. Plant Res. 7 (38): 2815-2822.

Bhuse, V.H., B.L. Lad, D.K. Patil, and V.M. Karade. 2003. Effect of time of planting, type of cutting and plant growth regulators on rooting in Pelargonium graveolens L. Herrit. Indian J. Agric. Res. 3:29-33

Boase, M.R., J.M. Bradley and N.K. Borst. 1998. An improved method for transformation of regal pelargonium (Pelargonium $x$ domesticum Dubonnet) by Agrobacterium tumefaciens. Plant. Sci. 139:59-69.

Brown, J.T. and B.V. Charlwood. 1986. The accumulation of essential oils by tissue cultures of Pelargonium fragrans (Willd). Fed. Europ. Biochem. Soc. 204 (1): 117- 120.

Desilets, H., Y. Desjardins and R. Belanger. 1993. Clonal propagation of Pelargonium x hortorum through tissue culture: Effects of salt dilution and growth regulator concentration. Can. J. Plant. Sci. 73: 871-878.

Dunbar, K.B. and C.T. Stephens. 1989. Shoot regeneration of hybrid seed geranium (Pelargonium $x$ hortorum) and regal geranium (Pelargonium $x$ domesticum) from primary callus cultures. Plant Cell, Tiss. Org. Cult. 19: 13-21.

Ghanem, S. A., U.I. Aly, A.A. El-kazzaz, A. Abdel-Samad and N.M. Arafa. 2008. In vitro regeneration of Pelargonium graveolens. J. Genet. Eng. Biotechnol. 6 (2): 15-18.

Gharib, F.A.L. 2005. Changes in regeneration and oil accumulation of Pelargonium nervosum under various culture condition. J. Bio. Sci. 5(5): 670-677.

Gupta, R., S.K. Gupta, S. Banerjee, G.R. Mallavarapu and S. Kumar. 2002. Micropropagation of Elit cultivars of rose- scented Geranium (Pelargonium graveolens L.' Herit.) for industrial production of propagules. Indian. J. Biotechnol. 1: 286-291.

Hassanein, A. and N. Dorion. 2005. Efficient plant regeneration system from 


\section{Rev. Fac. Agron. (LUZ). 2021, 38: 261-278. Abril-Junio.}

\section{Ebrahimzadeh et al.}

leaf discs of zonal (Pelargonium $x$ Hortorum) and two scented $(P$. capitatum and $P$. graveolens) geraniums. Plant. Cell. Tiss. Org. Cult. 83: 231-240.

Linsmaier, E.M., F. Skoog. 1965. Organic growth factor requirements of tobacco tissue cultures. Physiol. Plant. 18: $100-127$.

Murashige, T. and F. Skoog. 1962. A revised medium for rapid growth and bioassays with tobacco tissue cultures. Physiol. Plant. 15: 473-497.

Rao, P.V.L. 1994. In vitro plant regeneration of scented- leaved geranium Pelargonium graveolens. Plant. Sci. 98:193-198.

Saxena, G., S. Banerjee, L. Rahman, G.R. Mallavarapu, S. Sharma and S. Kumar. 2000. An efficient in vitro procedure for micropropagation and generation of somaclones of rose scented Pelargonium. Plant. Sci. 155: 133-140.

Skoog, F. and C.O. Miller. 1957. Chemical regulation of growth and organ formation in plant tissues cultured in vitro. Symp. Soc. Exp. Biol. 11: 118131.

SPSS software. 2012. SPSS user's guide, v. 21. IBM Corporation. New York, NY, USA.
Sukhumpinij, P., F. Kakihara and M. Kato. 2010. In vitro regeneration from mature leaf explants of Pelargonium rapaceum (L.) L'Hérit. Sci. Hortic. 126: 385-389.

Tembe, R.P. and M. Deodhar. 2010. Clonal propagation different cultivars of Pelargonium graveolens (L' Herit) viz Reunion, Bourbon and Egyptian. Biotechnol. 9 (4): 492-498.

Wojtania, A., E. Gabryszewska and A. Marasek. 2004. Regeneration of Pelargonium $\times$ hederaefolium 'Bonete' from petiole explants. Acta. Physiol. Plant. 26 (3): 255-262.

Yi QY, X., K.G. Jiang and M.H.T. Ren. 2010. Study on in vitro shoot culture of Pelargonium graveolens and its production. J. S. China. J. Agri. 2: 5967.

Zhou, J., G. Ma, E. Bunn and X. Zhang. 2007. In vitro shoot organogenesis from Pelargonium Citrosum Vanleenii leaf and petiole explants. Flori. Orna. Biotech. 1(2): 147-149.

Zuraida, A.R., M.A. Shukri, O. Ayu Nazreena and Z. Zamri. 2013. Improved micropropagation of biopesticidal plant, Pelargonium radula via direct shoot regeneration. Am. J. Res. Commun. 1(1): 1-12. 